\section{Setting the stage for cancer}

\section{By Lauren Martz, Staff Writer}

Although marketed blood-based diagnostics for leukemia are sufficiently sensitive to monitor minimal residual disease, no such tests exist for solid tumors. Now, German researchers have developed a personalized diagnostic that can stage solid tumors and detect minimal residual disease following therapeutic intervention. ${ }^{1}$ However, the approach requires the design of a unique assay for each patient, which may limit its commercial potential.

Many tumors are characterized by the presence of specific amplified genomic regions. Such regions have been reported to exist in $91 \%$ of breast cancers, $82 \%$ of glioblastomas and $75 \%$ of colon cancers. ${ }^{2}$

The sites at which single amplified genomic regions attach to each other are called amplicon fusion sites (AFSs). They are specific to individual tumors and detectable using PCR.

In a paper published in The Journal of Clinical Investigation, Axel Weber and colleagues at the University of Leipzig Children's Hospital reported a protocol that exploits the tumor specificity provided by AFS sequences to detect minimal residual disease in patients with solid tumors. The team's method involves sequencing the fusion sites from initial tumor samples and developing PCR primers to detect cells that have the same genetic characteristics as the primary tumors in unrelated organ and tissue samples from the same patient.

As proof of concept for the approach, dubbed AFS-PCR, the group took three neuroblastoma cell lines and 40 primary neuroblastoma samples with genomic amplifications near the gene MYCN ( $v$-myc myelocytomatosis viral related oncogene neuroblastoma derived; NMYC). The team used high-resolution tiling arrays, which include both coding and noncoding DNA, to map and sequence AFSs.

Using the AFS sequences, Weber's group designed PCR assays specific for each sample. In culture, the assays detected 1 MYCNamplified neuroblastoma cell in up to $8 \times 10^{6}$ neuroblastoma cells that lacked the amplification. The assays were specific for cancer cells with the same genomic amplifications as the primary tumors and did not generate any false positives when tested on control DNA isolates from human placental tissue.

In patient samples, the assays detected tumor cells in bone mar- row, peripheral blood and residual tumor tissue compared with no signal in control placental tissue.

"Once established from tissue of the primary tumor site, an individual AFS-PCR test offers a powerful tool to track tumor cells or tumor cell DNA in potential metastatic sites of any tissue origin as well as in bone marrow aspirates, in peripheral blood samples and even in cerebrospinal fluid, urine or any other sample of the patient," said Weber.

"AFS-PCR could significantly improve tumor staging at the time of initial diagnosis, the monitoring of a response to therapy by quantification of tumor cells in subsequent samples and the early detection of minimal residual disease or recurrent disease over time," added Weber, who is a medical doctor in the Department of Pediatric Oncology, Hematology and Hemostaseology at the hospital.

Howard Urnovitz, CEO and CSO of Chronix Biomedical, said diagnostics using AFS could provide more information about a given cancer than SNPs, which are germline biomarkers that might be more valuable for cancer risk assessment.

Chronix is running pilot trials of its breast and prostate cancer serum DNA tests, which detect cell-free circulating DNA associated with the cancers.

\section{(Individual) results may vary}

The AFS-PCR approach is broadly applicable to solid tumors, but two key drawbacks could impede the technology's commercialization.

First, the technique only is suitable for tumors with at least one identified amplified genomic region. Previous studies have shown that the majority of cancers contain amplified regions, but sequencing a patient's primary tumor, which is expensive, remains necessary to determine whether the regions are present.

Moreover, Weber noted that even if an amplified genomic region is present in a tumor, the ability to develop a tumor-specific assay isn't guaranteed.

"The method was described with highcopy amplified genomic regions," Weber said. While the technique should work in cancers with low copy numbers of amplified genome regions, he said "it can be difficult to identify a PCR primer" in such cases.

The second drawback is that the individualized nature of the test means an assay needs to be developed for each patient.

"What strikes me as not very pragmatic is that this strategy requires developing individual assays, which might not be able to be clinically employed and could be very expensive," said Achim Plum, SVP of corporate development at Epigenomics AG. "Using tiling arrays is a pretty costly approach."

Epigenomics markets Epi proColon, a PCR diagnostic that detects plasma levels of methylated septin 9 (SEPT9), a DNA methylation marker that is found in more than $90 \%$ of colon cancers and can be tested in routine blood plasma samples. 
Weber agreed that "at the moment, the costs of our procedure might be too high for comprehensive use." However, the cost of tiling arrays is coming down, which could make the method more affordable in the near future.

\section{"Using tiling arrays is a pretty costly approach." \\ -Achim Plum, Epigenomics AG}

minimal residual disease markers. Because no effective strategies are in routine use, we were not able to compare our strategy to others."

Plum acknowledged the potential for addressing an unmet need.

"If they are able to prove that this provides a significant medical benefit unable to be attained by available techniques, it could be possible that the cost and time investments are warranted," he said.

Weber said his team's next steps include an ongoing study of AFSPCR in a larger cohort of patients and studies to show that the ability to detect minimal residual disease provides information useful to the patients and clinicians. Specifically, he said the group needs to correlate patient prognosis with the presence of minimal residual disease detected by the method and show that the technique can be used for clinical decision making.

Weber said a patent application has been filed in Germany for the method and the IP is available for licensing.

Martz, L. SciBX 4(6); doi:10.1038/scibx.2011.154

Published online Feb. 10, 2011

\section{REFERENCES}

1. Weber, A. et al. J. Clin. Invest.; published online Jan. 10, 2011; doi:10.1172/JCI44415

Contact: Axel Weber, University of Leipzig Children's Hospital, Leipzig, Germany e-mail: axel.weber@medizin.uni-leipzig.de

2. Leary, R.J. et al. Proc. Natl. Acad. Sci. USA 105, 16224-16229 (2008)

COMPANIES AND INSTITUTIONS MENTIONED

Chronix Biomedical, San Jose, Calif.

Epigenomics AG (Xetra:ECX), Berlin, Germany

University of Leipzig Children's Hospital, Leipzig, Germany 